\title{
LA BRUTALITÉ PHOTOGRAPHIÉE
}

\section{Marin Dacos}

\section{Référence bibliographique}

Marin DACOS, “ La brutalité photographiée”, dans Frédéric CHAUVAUD (dir.), Violences. Société et représentation, $\mathrm{n}^{\circ}$ 6, Credhess, Paris, juin 1998, pp. 103-114.

\section{Résumé}

La photographie révèle un regard qui montre l'historicité des représentations de la violence. Dans le premier quart $\mathrm{du} \mathrm{XX}^{\mathrm{e}}$ siècle, la tuerie que notre société s'applique à dissimuler derrière des murs et des euphémismes, n'est encore qu'une pratique commune qui ne pose pas de problème de conscience. Théophile Magné, artisan passionné de techniques, a voulu immortaliser la tuerie, en lui ajoutant le piment de la féminité. Emile Establet, paysan de la plaine comtadine, confronté à la Grande Guerre, voit la violence d'un autre œil. Elle est avant tout désolation et tristesse. Les terres sont ravagées, les hommes morts ou blessés. Son appareil photographique en dit plus sur l'horreur de la guerre que son carnet, dans lequel il ne s'épanche pas.

\begin{abstract}
Photography reveals a way of looking which points out the historical feature of representations of violence. In the first quarter of the twentieth century, the slaughtering of animals that our society took pains to hide behind walls and euphemisms, was still just a common practice which did not pose the problem of conscience. Théophile Magné, a craftsman fascinated with various techniques wanted to immortalise the slaughtering by adding to it the spice of femininity. Emile Establet, a peasant from the plain of Vaucluse, who was confronted with the First world war, saw violence from another point of view. It was before all desolation and sadness. Lands where devastated, men dead or wounded. His camera spoke more about the horror of the war than his notebook, in which he did not poor out his feelings.
\end{abstract}


L'étude historique du regard photographique participe à l'histoire culturelle dans la mesure où elle s'intéresse à la production et à la consommation d'un objet culturel, l'image photographique. Elle se situe à la croisée de l'histoire des goûts, des processus d'influence culturelle et de "l'histoire des choses banales" chère à Daniel Roche. Appliquée à la photographie amateur, elle révèle la mise en scène du quotidien et la construction volontaire d'une mémoire. Point de vue sur la perception du paysage et des petits instants de la vie, l'image photographique révèle un regard dont l'étude reste à faire. ${ }^{1}$ Etudié de préférence dans l'univers rural vauclusien, où l'image est relativement rare au début du siècle, le regard photographique ne révèle pas en priorité le quotidien laborieux des hommes de la terre, mais, semble-t-il, plus souvent le portrait des proches du photographe ainsi que les événements majeurs de la vie, comme le mariage. Dans ce contexte, la violence prend a priori peu de place. Cependant, dès 1914, la guerre sépare les familles et bien souvent les photographes de leur entourage. Se retrouvant face aux lignes allemandes, dans un univers dont la violence extrême n'est pas à démontrer, l'amateur photographe pourrait être tenté par le silence photographique. Il n'en est rien. De l'image familiale paisible aux photographies de femmes abattant des animaux jusqu'aux clichés douloureux de la Grande Guerre, le regard photographique se révèle complexe. La violence y prend des formes sous-jacentes, banales ou démesurées. $^{2}$

${ }^{1}$ Marin DACOS, "L'œil et la terre. Vers une histoire du regard (1900-1950)", Ruralia, n ${ }^{\circ}$, Paris, Association des ruralistes français, 1997, pp. 34-64.

${ }^{2}$ La démarche s'appuie sur l'étude de trois corpus très différents, se situant entre 1910 et 1925 , qui servent de fil directeur au propos sans prétendre être représentatifs. Seul l'élargissement du corpus pourra apporter quelques éléments à portée plus générale. 


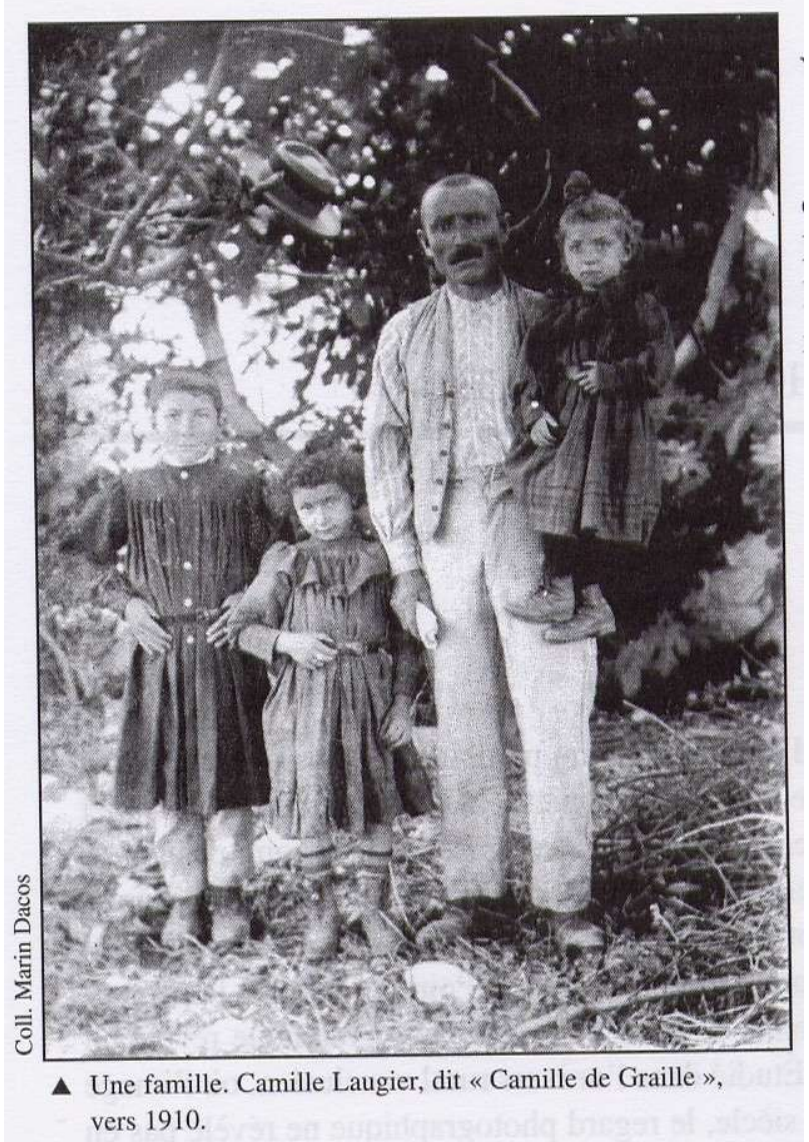

Photographie $n^{\circ} 1$. Une famille. Camille Laugier, dit "Camille de Graille", vers 1910.

\section{Violence et quotidien}

L'homme a posé son chapeau dans les arbres derrière lui. Il porte une petite fille dans les bras comme si elle ne pesait rien. A sa droite, deux autres enfants. Vers 1910, le sourire n'est pas encore entré dans les mœurs photographiques de ces habitants du village de Méthamis (Vaucluse) : il faut être digne devant l'appareil, la petite famille côtoie l'éternité. Cette image, comme les albums photographiques vauclusiens élaborés entre 1900 et 1950, montre le caractère paisible de l'image familiale et souligne le paradoxe qui consiste à chercher de la violence dans ce type de documents. Que ce soit dans les albums des familles Février, Burgues, Bonneau, Camille “de Graille”, la violence n'est pas présente. Une lecture attentive ne parvient pas à déceler une image d'accident, de dispute, de décès ou de violence sur un animal. Peut-il en être autrement, dès lors que la fonction première de l'image familiale est la constitution consensuelle d'une mémoire collective destinée à asseoir l'unité du groupe ? D'une manière générale, la violence n'est pas considérée comme photographiable ${ }^{3}$, mais des

\footnotetext{
${ }^{3} \mathrm{Au}$ début des années 1960 une enquête demandant de classer les thèmes pouvant donner lieu à une belle image donne les résultats suivants. En tête de liste : un coucher de soleil, un paysage, une fillette jouant avec un chat ; aux quatre dernières places figure : une querelle de clochards, un étal de boucher, un homme blessé et un accident de voiture. Pierre BOURDIEU (Dir), Un art moyen. Essai sur les usages sociaux de la photographie, Paris, Editions de Minuit, 1965, p. 130-131.
} 
exemples montrent que les scènes d'accident ne sont pas exclues du champ photographique. Ainsi, il est possible de photographier l'accident d'un tramway ${ }^{4}$, ou celui d'une voiture de course. ${ }^{5}$ Mais dès lors, la photographie sort du champ familial, et s'inscrit radicalement dans l'espace public. Même l'ex-voto représente relativement peu d'accidents, alors que l'issue heureuse de l'événement pourrait faciliter sa représentation. ${ }^{6}$ En tant qu'instrument de mémoire, la photographie familiale est plus intimement liée à la mort qu'à la violence physique. ${ }^{7}$ Cette logique est visible en négatif, à travers l'absence ou l'extrême rareté de la mort dans les albums familiaux. ${ }^{8}$

La violence n'est pas toujours à l'intérieur de l'image ; par sa rareté, ou par son objet, l'image elle-même peut devenir le signe ou le vecteur d'une violence moins évidente. Il faut notamment s'interroger sur la nature de la première rencontre entre l'image photographique et l'individu au début du siècle. L'impression de réalité et même de danger provoquée par les premières projections cinématographiques du train entrant en gare de La Ciotat est célèbre, mais qu'en est-il de la première image de soi ? Le premier contact peut être marqué par une frustration, par le sentiment d'être exclu du monde de l'image. Ce type de réaction est devenu très rare de nos jours ${ }^{9}$ car les blocages techniques et financiers sont désormais minimes. Mais, tant qu'une évaluation sérieuse de la diffusion sociale des appareils photographiques ne sera pas réalisée, il est permis de penser que la photographie reste cantonnée socialement au début $\mathrm{du} \mathrm{XX}^{\mathrm{e}}$ siècle, et suscite des ressentiments. Ceux qui possèdent le privilège photographique, en particulier à la fin du $\mathrm{XIX}^{\mathrm{e}}$ siècle, ne mettent-ils pas en œuvre une violence parallèle en photographiant ceux qui ne disposent pas d'appareil ? En particulier, les pratiques liées aux travaux de Bertillon sur l'identité, peuvent relever du vol de l'image de l'autre. ${ }^{10} \mathrm{Un}$ processus similaire se produit lorsque la photographie, à la suite de la peinture, achève de désamorcer la peur provoquée par les paysages tourmentés. ${ }^{11}$ L'appareil photographique devient dès lors l'attribut d'une réelle conquête dont la violence s'apparente à une prise de possession voire à

\footnotetext{
4 Jacques FILHOL, Mémoire de l'oubli. La photographie populaire en Charente. 1880-1920, Musée municipal d'Angoulême, 1983, image $\mathrm{n}^{\circ} 109$.

${ }^{5}$ Jacques FILHOL, As du volant et chauffeurs du dimanche, Sans lieu d'édition, Le montreur d'images, s.d., p. 30-31.

${ }^{6}$ Bernard COUSIN, Le miracle et le quotidien. Les ex-voto provençaux, images d'une société, Aix, 1981, $339 \mathrm{p}$.

${ }^{7}$ Régis DEBRAY, Vie et mort de l'image. Une histoire du regard en Occident, Paris, Gallimard, 1992, p. 53

${ }^{8}$ Cf. la photographie de la dépouille mortelle de Victor Hugo : André JAMMES, Nadar, Paris, Centre national de la photographie, 1989, image $\mathrm{n}^{\circ} 61$. L’image d'un enfant sur son lit de mort est-elle exceptionnelle ? Cf. Jacques FILHOL, Mémoire de l'oubli. ouv. cit., image n89. La photographie a été prise par un photographe professionnel.

${ }^{9}$ Tout au plus est-il restreint au Quart-Monde.

${ }^{10}$ Voir à ce sujet les astuces proposées par Bertillon en 1890 pour obtenir de son sujet une pose naturelle dans Michel FRIZOT, Françoise DUCROS, Du bon usage de la photographie. Une anthologie de textes, Paris, Centre national de la photographie, 1987, p. 102.

${ }^{11}$ J.R. SOUBIRAN, Le paysage provençal et l'école de Marseille avant l'impressionnisme, 1845-1874, Toulon, Réunion des musées nationaux, Musée de Toulon, 1992, 617 p.
} 
une pacification. Dominique Piazza, qui associe photographie et excursions, ne déroge pas à la règle. ${ }^{12}$ Les photographies coloniales relèvent également de la prise de contrôle d'un espace inconnu. De Bertillon à la Salpêtrière, de l'image coloniale à la photographie provençale, le monde exotique ou hors-normes est mis en boîte, sagement recensé, étiqueté, en quelque sorte apprivoisé. Ce mécanisme concurrence les populations qui sont dépossédées de leur paysage ou de leur image. La réaction hostile des habitants du petit village de Méthamis à l'arrivée de Frédéric Mistral ${ }^{13}$ montre que les excursionnistes sont perçus comme des étrangers qui traversent un territoire sur lequel ils n'ont rien à faire. L'accueil glacial qui est réservé à ces messieurs de la ville est à la mesure de l'agression que représente leur intrusion dans le territoire communal.

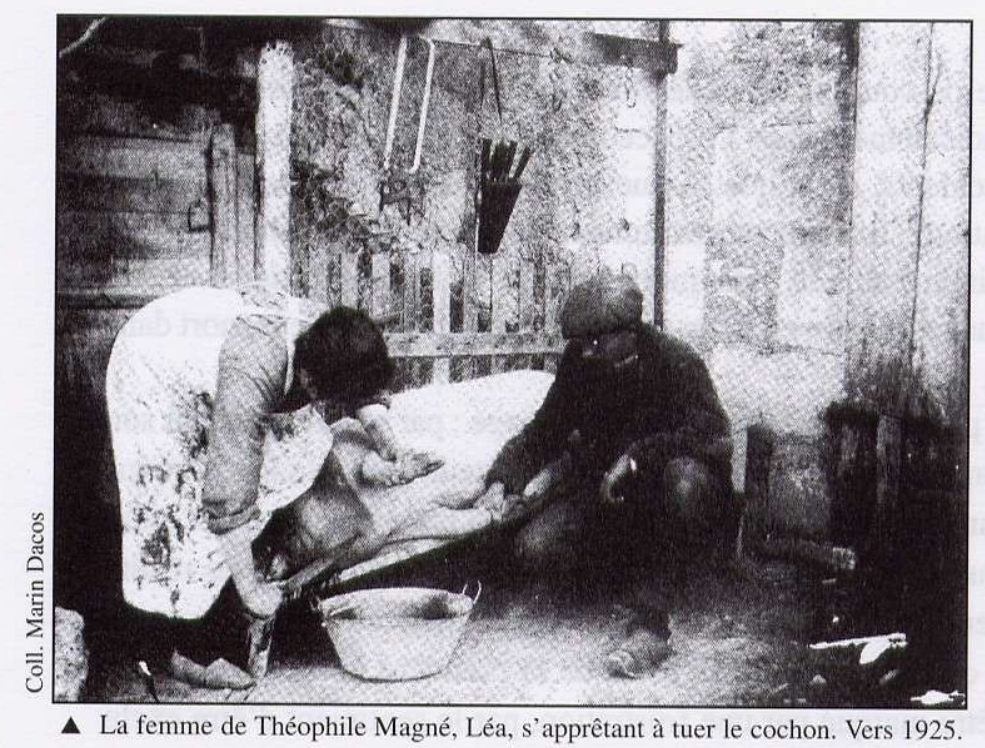

\section{Photographie $n^{\circ}$ 2. La femme de Théophile Magné, Léa, s’apprêtant à tuer le cochon. Vers 1925.}

Le moment est crucial. La lame s'apprête à faire passer le cochon de vie à trépas. La femme qui tient l'imposant couteau a un tablier taché de sang : ce n'est pas la première fois qu'elle procède à la tuerie d'un animal. Un homme en sabots, accroupi, tient une patte du cochon. La bassine est posée à l'endroit même où jaillira le sang versé. Il ne faut rien perdre. L'homme qui a pris cette photographie, Théophile Magné ${ }^{14}$, menuisier à St-Quentin de

Serge BRIFFAUD, Naissance d'un paysage. La montagne pyrénéenne à la croisée des regards. XVIe XIXe siècle, Toulouse, AGM-AHP, 1994, $622 \mathrm{p}$.

Marin DACOS, "L'image de la Nesque au XIXe siècle. La Nesque des légendes", Les Carnets du Ventoux, Avignon, A. Barthélemy, Déc. 1994, pp. 95-101.

Marin DACOS, "L'image de la Nesque au XIXe siècle. La découverte de la Nesque", Les Carnets du Ventoux, Avignon, A. Barthélemy, Juin 1995, pp. 100-105.

${ }^{12}$ Le pionnier de la carte postale photographique fut président des Excursionnistes marseillais. Cf. Isabelle RAMBAUD, Jean-Claude BOUZE, Dominique Piazza 1860-1914 inventeur de la carte postale photographique, ou l'image avant la lettre, Marseille, Archives de la ville de Marseille, 1991, 46 p.

${ }^{13}$ Frédéric MISTRAL, Mémoires et récits, Raphaèle les Arles, Marcel Petit éd., 1981, pp. 671-683.

${ }^{14}$ Sauf indication contraire, toutes les informations proviennent de Jacques FILHOL, A la guerre comme à la guerre. Clichés sur verre de Théophile Magné (1881-1964), Sans lieu d'édition, Le montreur d'images, s.d., 24 p. En revanche, sauf indication contraire, les interprétations me sont personnelles. 
Chalais (Charente), a laissé 134 clichés ${ }^{15}$ dont la plupart sont sans surprise, mais dont cinquante trois exemplaires manifestent une réelle originalité. Ces images montrent en effet des femmes tuant des animaux, et pas seulement des bêtes de petite taille. Quelle est la motivation de ces images extraordinaires, de femmes saignant un lapin, une oie, un chevreau, dépeçant un cochon, mettant à mort un veau après l'avoir solidement ligoté, ou égorgeant un bœuf couché au sol ? Jacques Filhol a cru voir dans ces images un témoignage de la guerre des femmes entre 1914 et 1918, à l'arrière. Cette interprétation ne parvient pas à épuiser la richesse et l'originalité de la démarche. Jacques Filhol lui-même, reconnaît que la chronologie infirme en partie sa thèse. La série semble en effet s'étendre sur quinze années, de 1910 à 1925, ce qui marque une détermination continue de la part du photographe, mais surtout extrait la série du contexte direct de la guerre.

L'image, confuse, de la femme de Magné découpant de la viande dans une chambre, à côté d'un lit dont les draps sont défaits, et face à un mur auquel est accroché un chapelet ${ }^{16}$, peut faire pencher pour une interprétation intimiste, voire sexuelle, de la série. Théophile Magné serait-il fasciné par l'image de sa femme pataugeant dans le sang ? Cette hypothèse n'est que peu vraisemblable car Magné a photographié sa femme Léa, mais également sa bellemère Rosa, sa belle-sœur Aglaé, son neveu Léandre, sa nièce Rachel, ses sœurs Maria et Rachel... ainsi que d'autres femmes dont l'identification n'est pas possible. L'hypothèse d'une fascination esthétisante pour la violence, proche du mouvement futuriste ${ }^{17}$, n'est pas beaucoup plus vraisemblable, ne serait-ce qu'en raison de la sobriété de la mise en scène. Les postures sont dignes et modestes, même si le regard n'évite pas toujours l'objectif. Les yeux des femmes sont le plus souvent rivés sur leur travail, ignorant le photographe. Le couteau, présent dans neuf clichés sur dix, bien en évidence mais jamais montré ostensiblement, n'apparait pas comme une arme mais comme un outil.

C'est en effet probablement l'hypothèse artisanale et technique qui rend le mieux compte de la démarche de Théophile Magné. Menuisier, il s'est fait représenter à deux reprises travaillant à son établi ${ }^{18}$ tandis qu'il a photographié le maréchal ferrant au travail. ${ }^{19}$ Passionné par la technique, il possède un appareil photographique, porte une grande attention à construire ses clichés ${ }^{20}$, et surtout photographie des objets et des gestes techniques. Il a notamment photographié une voiture, un avion, un enfant "essayant une petite machine à

\footnotetext{
${ }^{15}$ Jacques FILHOL, Mémoire de l'oubli. La photographie populaire..., ouv. cit., p. 29.

${ }^{16}$ Jacques FILHOL, A la guerre comme à la guerre..., ouvr. cit., p. 10.

${ }^{17}$ Fanette ROCHE-PEZARD, L'aventure futuriste 1906-1916, Paris, EFR, 1983, 505 p.

18 Jacques FILHOL, Théophile Magné (1881-1964), menuisier et photographe, Sans lieu d'édition, Le montreur d'images, s.d., page de couverture, et page 24 .

${ }^{19}$ Ibidem, p. 25

${ }^{20}$ Ainsi, la construction d'une image présentant deux jeunes filles marchant d'un pas alerte sur une plage est-elle remarquable. Les filles sont cadrées sur la gauche de l'image alors qu'elles se dirigent vers la droite, laissée quasiment vide.
} 
vapeur" ainsi qu'une locomotive, en faisant poser les cheminots près de l'énorme engin. Ses clichés de femmes tuant les bêtes ne sont rien d'autre que la reconnaissance d'une technique maîtrisée, doublée sans doute du paradoxe résidant dans l'association entre la mort et celles qui donnent la vie. A la manière d'un reportage, quand il le peut — mais les plaques sont chères —, il s'offre le luxe de photographier la succession des étapes de la mise à mort ou du travail sur la dépouille. Dès lors, l'image de groupe représentant tous sexes confondus ces professionnels de la mort dans leur lieu de travail -un abattoir- prend sens, en rendant hommage aux artisans. La banalité de ces scènes dans le monde rural explique probablement leur rareté dans les lots vauclusiens. Théophile Magné a peut-être senti que les temps changeaient, que les abattoirs industriels allaient remplacer la tuerie domestique, et qu'il y avait là une technique originale et digne d'image. Tout ceci explique que la violence objectivement représentée n'est probablement pas perçue comme telle par l'auteur des images. Elle n'est pour lui que le tableau d'un moment important de la vie quotidienne. La présence fréquente du sang, sur les tabliers, dans les bassines et même en train de couler du cou d'une oie, marque un rapport daté avec les liquides du corps ${ }^{21}$, que l'on désire aujourd'hui cacher dans les abattoirs. Théophile Magné témoigne d'une mentalité intermédiaire entre le temps où la scène de tuerie est banale et publique, et celui où règne la dissimulation de la mort animale par des murs, la déresponsabilisation de l'acte par taylorisation du travail d'abattage, la dissimulation du sang par euphémisation des représentations iconographiques. ${ }^{22}$ En somme, l'œil de nos contemporains voit de la violence là où la rétine de Théophile Magné n'enregistre que des pratiques. La violence est beaucoup moins ambiguè au cœur des tranchées, sous les obus et dans le silence de l'attente.

\footnotetext{
${ }^{21}$ Alain Corbin parle de "retrait du spectacle de l'épanchement du sang" dans Alain CORBIN, "Le sang de Paris. Réflexions sur la généalogie de l'image de la capitale", Le temps, le désir et l'horreur, Paris, Aubier, 1991, p. 219.

${ }^{22}$ Maurice AGULHON, "Le sang des bêtes. Le problème de la protection des animaux en France au XIXe siècle", Romantisme, Nㅜ 31, Paris, 1981, pp. 81-109. Egalement, Noélie VIALLES, Le sang et la chair. Les abattoirs du pays de l'Adour, Paris, Maison des sciences de l'homme, 1987, p.24. L'auteur cite l'article "abattoir" dans les éditions successives des encyclopédies Larousse. En 1905 des dessins présentent en détail étapes et outils. En 1928, la modernisation est déjà en route avec l'ajout d'illustrations sur les abattoirs de Chicago. Enfin, en 1982, toute illustration réaliste est abandonnée au profit de schémas décharnés.
} 


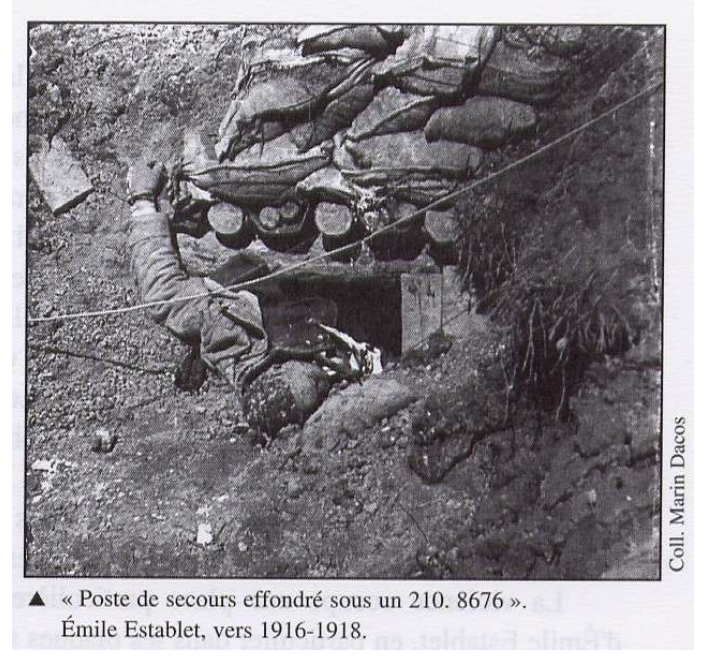

\section{Photographie n’3. “Poste de secours effondré sous un 210.8676 ”. Emile Establet, vers 1916-1918.}

\section{Le silence et l'image, regards sur la Grande Guerre}

"Poste de secours effondré sous un 210". La description est brève, et ne saurait se comprendre sans l'image qui lui est associée. Du cadavre enseveli sous ces kilos de poutres, de pierres et de terre, émergent péniblement une tête, une épaule, un bras. L'homme a sans doute été surpris par l'effondrement alors qu'il tentait de sortir de son abri subitement transformé en poids mortel et en tombeau. Il est resté saisi dans ce dernier mouvement désespéré pour sortir à l'air libre. ${ }^{23}$ Le déchaînement de violence n'a pu être photographié sur le vif, mais le photographe a pris une image qui en porte les stigmates. Il n'y a dans cette démarche photographique macabre rien d'évident, rien de facile à interpréter. En dehors des photographes professionnels qui ont toujours offert aux événements guerriers et révolutionnaires une place de choix ${ }^{24}$, les hommes de troupe engagés dans le conflit portent un regard plus intime sur la violence guerrière. Le cas d'Emile Establet, “propriétaire " à Sorgues (Vaucluse), agriculteur dynamique de la plaine comtadine, n'est pas isolé et mériterait d'être comparé avec d'autres albums de poilus. Musicien, brancardier, et photographe pour l'armée, Emile Establet a été incorporé en $1912^{25}$ et n'a quitté l'habit militaire qu'en 1918, sérieusement blessé aux poumons par des éclats d'obus. Il a laissé deux séries d'images de la Grande guerre. Une première série est connue sous la forme de 104 tirages sur papier conservés dans des boites et dans un album de famille, composé au soir de sa vie. ${ }^{26} \mathrm{La}$

\footnotetext{
${ }^{23}$ La datation de l'image n'est pas facile, mais on peut exclure l'année 1915.

${ }^{24}$ De la guerre de Crimée à la Première guerre mondiale, de la Commune à la guerre d'Espagne. Cf. par exemple François ROBICHON, André ROUILLÉ, Jean-Charles LANGOIS, La photographie, la peinture, la guerre. Correspondance inédite de Crimée (1855-1856), Nîmes, Ed. J.Chambon, 1992,310 p.

${ }^{25}$ Selon toute vraisemblance.

${ }^{26}$ Né en 1891, il décède en 1978. Les albums ont été élaboré vers 1976-1977, avec des tirages datant selon toute vraisemblance de l'immédiat après-guerre. Un seul album (l'album bleu) concerne le premier conflit
} 
deuxième série a été conservée sous la forme de 49 plaques positives stéréoscopiques. ${ }^{27}$ Emile Establet a donc disposé, au cours du conflit, de quatre sources d'images. Dans un premier temps, il semble ne pas posséder d'appareil. Il profite de photographes de passage et reçoit des images par courrier. Le 1er Janvier 1915, il note dans son carnet: "Noèl et Achille (...) se font photographier après-midi et je reçois photo Marie-Thérèse et lettre parents ". ${ }^{28}$ Ensuite, il demande un appareil photographique à sa famille ${ }^{29}$ qu'il ne reçoit pas avant la fin du carnet, le 16 février 1915. Enfin, semble-t-il vers 1916, il devient photographe pour l'armée et ce au moins jusque $1917 .{ }^{30}$ Les toponymes mentionnés sur les plaques permettent d'identifier au moins 18 images de Verdun. Plus de quarante ans après la guerre, Emile Establet a montré ces images à ses petits-enfants, mais rien ne prouve qu'il ait produit ces images dans le but d'en tirer une leçon sur la guerre, et il faut se méfier de lectures a posteriori de la guerre.

La violence occupe une place particulièrement importante dans le regard d'Emile Establet, en particulier dans les plaques stéréoscopiques. Sur les 49 plaques, plus de la moitié présentent des scènes annonçant et surtout suivant la violence : quatorze présentent des paysages détruits ou désolés, neuf montrent des cadavres, quatre des blessés et deux des scènes de guerre (bombardements, hommes montant à l'attaque). A cela s'ajoutent les deux images directement liées à la mort, représentant des croix. La vie des poilus, avec huit images, n'est pas réellement négligée, d'autant plus qu'elle occupe une grande place dans les images de la série beaucoup plus personnelle de l'album bleu. La dominante de paysages dévastés se retrouve d'ailleurs sur les tirages papier, comme par exemple les images sous-titrées "Belgique. Ferme de statuelle" et "Ici était un village" représentant des paysages ravagés par la guerre, dans lesquels la présence passée d'une ferme ou d'un village est difficilement imaginable. Cette fascination pour la blessure qu'impose la guerre au paysage, qui se retrouve sous la plume des soldats ${ }^{31}$, s'explique-t-elle, au-delà de sa force cathartique, par le fait que le

mondial. Les autres clichés, non militaires, datant d'avant et surtout d'après la guerre, ne sont pas pris en comtpe. Merci à ses petits enfants pour m'avoir ouvert la porte de leurs archives et avoir contribué à ma connaissance de cet homme.

${ }^{27}$ Il est probable que les deux séries aient été prises par des appareils différents, mais rien n'indique que leur auteur soit quelqu'un d'autre qu'Emile Establet. La concordance des lieux entre les deux séries, visible sur les images, mais également dans les commentaires écrits manuellement sur les plaques et dans l'album, vont dans le sens de l'unification du corpus.

${ }^{28}$ Collection particulière.

${ }^{29}$ Le 2 décembre 1914, il écrit dans son carnet: “Je fais la demande 1 appareil photo". Le 14 décembre, "Je reçois 4 lettres 2 Paul (...) une carte de Léonie (...) et une lettre M [sa future épouse] réponse à l'appareil photo " (collection personnelle).

${ }^{30}$ La seule plaque datée porte la mention “ 1917 ”. Il est possible également que ces images aient été vendues par leur auteur à la presse d'illustration comme le fit Blaise Cendrars. Cf. Laurent VERAY, "Montrer la guerre : la photographie et le cinématographe", dans Jean-Jacques BECKER et alii, Guerres et cultures, Paris, Armand Colin, 1994, pp. 233.

${ }^{31}$ Stéphane AUDOIN-ROUZEAU, 14-18, les combattants des tranchées à travers leurs journaux, Paris, Armand Colin, 1986, p. 88.

Gérard CANINI, Combattre à Verdun. Vie et souffrance quotidiennes du soldat 1916-1917, Paris, Presses universitaires de Nancy, 1988, p. 41-44. 
photographe est également paysan ? L'école de la République lui a appris la belle diversité des paysages de France, et son activité agricole l'a rendu sensible au prix d'une terre travaillée, amendée, sans cesse mise en valeur par le travail de l'homme. Il ne faut cependant pas exclure les raisons purement conjoncturelles. Un brancardier peut-il s'interrompre pour photographier un cadavre ? A-t-il son appareil sur lui ? Il est plus probable qu'Emile Establet ait produit l'essentiel de ses clichés à ses heures perdues, ce qui explique sans doute qu'il rencontre, à cet instant, moins de morts que de paysages. Enfin, dans la longue attente qui est celle du soldat dans les tranchées, que faire d'autre sinon regarder autour de soi, derrière et devant, à l'Est et à l'Ouest, et constater que la réalité du front est constituée de ces paysages irréels que l'on a souvent qualifiés de lunaires, comme s'ils appartenaient à un autre monde?

Les neuf images de cadavres sont photographiées très froidement, et décrites avec la même avarice de mots que le "Poste de secours effondré sous un 210", ainsi l'image de cadavres dans une tranchée vue en coupe est-elle nommée "Tranchée de départ", et l'image d'un cadavre sur le dos couché au sommet d'une tranchée, la tête renversée et le visage caché, porte-t-elle le laconique : "Tranchée de départ d'attaque". Ce n'est pas tant la place limitée - qui manque ${ }^{32}$, ce sont les mots qui font défaut, la souffrance qui rend muet. Dans l'image d'une jambe arrachée comme surgie du néant, Emile Establet ne parvient pas à décrire ce qu'il voit, et il inscrit un mystérieux "Près du fort de Douaumont". Dans son carnet, les allusions à la violence sont très rares, et souvent brèves comme le 14 décembre 1914 : " Un aéroplane a jeté une bombe sur Ambréville tuant 1 artilleur et en blessant 2 autres ", le 27 décembre 1914 : "la fusillade est terrible du côté du 5eme corps", et, le plus long des commentaires du carnet : " 3 soldats dont 1 lieutenant du 141 se sont fait écraser par le train la nuit derrière en venant choisir leur cantonnement. Les trains ne marchent que la nuit et sans lanterne de crainte $d u$ bombardement.". ${ }^{33}$ La guerre est indicible, seul peut-être l'appareil photographique parvient à révéler l'émotion du soldat, à matérialiser un instant son angoisse dans une image.

Le terme "blessé" ne gêne pas Emile Establet, mais il ne parvient à utiliser le mot "cadavre" que pour les dépouilles mortelles allemandes : les cadavres français sont presque toujours ignorés dans la description de l'image, au profit d'une localisation géographique anonyme. Est-ce à dire qu'Emile Establet traite différemment les Allemands des Français ? L'étude des images de la violence guerrière ne peut faire l'économie d'une approche de la perception de l'ennemi, basée notamment sur la comparaison entre la photographie produite

${ }^{32}$ Sur une autre plaque Emile Establet a su ajouter une ligne complémentaire dans l'espace réservé au positif.

${ }^{33}$ Le 22 novembre 1914. Collection particulière. 
par les poilus avec les caricatures illustrées produites par la presse. ${ }^{34}$ Les exactions allemandes ${ }^{35}$ du début de la guerre, dont la connaissance circule vite, nourrissent l'image du Boche qui s'élabore depuis la défaite de 1870. Le dessin de caricature véhicule des images associant les Allemands à l'excrément, inversant les fesses et le visage, et fondant des stéréotypes physiologiques : le gros soldat prussien avide et pillard, gavé de choucroute et de bière ; l'officier prussien filiforme, cruel et arrogant. La carte postale, elle aussi, véhicule des stéréotypes dégradants, comme "Papa ne fait pas de grâce aux Prussiens. Maman dit qu'ils ne valent pas les chiens". ${ }^{36}$ On pourrait s'attendre à voir ces stéréotypes influencer le simple soldat brancardier qu'est Emile Establet. En réalité, seules cinq plaques représentent des Allemands, ce qui correspond à moins du dixième de l'ensemble. Cette relative rareté s'explique aisément par les contraintes de la guerre. Deux de ces images présentent les constructions ennemies, une autre un blessé allemand, et deux dernières des cadavres allemands. Ici, contrairement à ce que constate Laurent Veray dans la presse de l'arrière, les Allemands meurent apparemment moins que les Français ${ }^{37}$, puisque dans le même temps cinq cadavres et cinq blessés français sont photographiés. Emile Establet voit les siens tomber en plus grand nombre, par le simple effet de la proximité, et il n'a pas les préoccupations des rédacteurs de L'Illustration. Si cela prouve qu'il n'a pas de souci de propagande ou de censure, cela ne répond pas à la question de sa perception de l'ennemi. La comparaison du traitement photographique des cadavres ne montre pas de différence notable entre les morts de chaque camp. La froideur des images de cadavres, tant allemands que français, semble être le signe d'un profond respect de la mort. Ainsi, l'image "Champ de bataille des Caurières" montre le cadavre d'un homme blessé dont le torse est bandé, allongé au pied d'un arbre, semblant glisser au milieu des cailloux, la tête tournée vers le bas. Les images de "Cadavres allemands" présentent des corps repliés sur eux-mêmes, gisant sur le sol. Il est difficile de déceler une différence de regard photographique sur l'un ou l'autre cadavre : la distance au cadavre est toujours grossièrement la même - la plus grande proximité étant réserve à une jambe arrachée-, le traitement des visages, très anonyme dans tous les cas, ne semble pas varier sensiblement, et surtout aucune mise en scène n'est réalisée. Les cadavres français ne sont pas rétablis dans une dignité que leur situation leur retire ${ }^{38}$, les cadavres allemands ne sont pas exhibés comme des trophées. Il n'y a pas de mimique vengeresse, pas de mise en scène macabre pouvant ressembler à certaines images prises par les nazis durant la Deuxième guerre mondiale. Pourtant, Emile Establet a le goût de la mise en scène, lorsque celle-ci s'impose

\footnotetext{
${ }^{34}$ Ouriel RESHEF, Guerres, mythes et caricatures, Au berceau d'une mentalité française, Paris, Presses de la fondation nationale des sciences politiques, 1984, $230 \mathrm{p}$.

35 Jean-Jacques BECKER, Stéphane AUDOIN-ROUZEAU, La France, la nation, la guerre : 1850-1920, Sedes, Paris, 1995, p. 295

${ }^{36}$ Stéphane AUDOIN-ROUZEAU, 14-18, les combattants..., ouv. cit., p.152.

37 Laurent VERAY, “Montrer la guerre : la photographie et le cinématographe ”, dans Jean-Jacques BECKER et alii, Guerres et cultures, Paris, Armand Colin, 1994, pp. 234.

${ }^{38}$ Laurent Veray note une propension de la presse à photographier des croix et des tombes du côté français, et des cadavres du côté allemand afin d'atténuer l'impact des images des morts françaises.
} 
pour traduire son état d'esprit et celui de ses camarades. ${ }^{39}$ Les heures sont trop graves pour permettre qu'une image ressemble à celle prise en temps de paix par Léopold Dussouchet le 13 Août 1908, sur laquelle les soldats portent fièrement à la main des boulets de canon, avec une pancarte explicite : "Dragées pour Guillaume ". ${ }^{40}$

Enfin, il faut revenir sur la différence de traitement entre Français et Allemands dans le titre inscrit sur la plaque, dans l'interstice qui sépare la vue gauche de la vue droite. Outre la distinction déjà signalée dans l'usage du mot "cadavre", une double vision de l'ennemi semble s'ancrer sur des éléments visuels différents. Ainsi, les constructions sont-elles " boches" et les cadavres "allemands": "Lignes françaises et boches", " Abris boches devant Noordschood", "Douaumond. Blessé allemand ", "Cadavres allemands". ${ }^{41}$ Peut-on voir là la distinction entre un ennemi lointain profondément haï, incarné par les bâtiments, globalement désigné par le très péjoratif “boche ", et un ennemi humain, individualisé, qui fait lui aussi partie des victimes de la guerre, qualifié du terme plus neutre "d'allemand"?

En insistant sur les aspects violents, le danger existe de négliger la profonde dichotomie qui se manifeste dans le regard d'Emile Establet. Témoin de la guerre, touché par les destructions de terres, par les hommes blessés ou tués, il poursuit malgré tout sa vie. De nombreuses images témoignent de ce quotidien, comme ces images de l'album bleu: " $A u$ repos cachet (sic) dans la forêt", "Violons et violoncelles fabriqués par Jacquier en récupérant de vieilles planches", ou celles des plaques stéréoscopiques : "Abri de la cote de Froideterre", “Jomilly. Ravitaillement". Il y a bien là une confrontation surréaliste entre deux niveaux de réalité, la violence par essence exceptionnelle, même si elle est quotidienne, et le quotidien, par essence commun, même si parfois il devient rare.

\footnotetext{
${ }^{39}$ Deux images de l'album bleu montrent son escouade de musiciens grimés et rieurs devant l'objectif (images non datées).

${ }^{40}$ Jacques FILHOL, Léopold Dussouchet (1887-1963), photographe ambulant, Sans lieu d'édition, Le montreur d'images, s.d., p. 6

${ }^{41}$ Je souligne.
} 
Alors qu'au loin résonnaient le bruit des canons et les cris des hommes tombés sous la mitraille, partout en France, et jusque dans les tranchées, il fallait bien vivre. Les années 1910 et 1920 sont marquées par ce profond contraste qui fait cohabiter violence et vie quotidienne. Avant la guerre, la photographie semble n'avoir de violence que symbolique, dans la capture de l'image de l'autre, ou dans la valorisation exclusive de certaines parties de la population par la chambre noire. La violence quotidienne dans l'image, rare, ne relève pas de la sphère privée mais publique. En cela, elle dissimule la première des violences, celle qui se loge dans la maisonnée, et participe au processus d'apprivoisement de la violence relevé notamment par Frédéric Chauvaud. ${ }^{42}$ Théophile Magné, photographe de femmes tueuses d'animaux, en représentant la technique artisanale plus que la violence d'une tuerie qu'il n'appréhende pas comme telle, ne vient pas contredire ce schéma. C'est la Grande Guerre qui change tout, en marquant une rupture fondamentale dans la vie quotidienne des Français et dans la pratique de la guerre. ${ }^{43}$ Pour supporter la brutalization, pour survivre à l'enfer, le menu du repas du soir occupe plus de place dans les écrits d'Emile Establet que les récits de violence. C'est son appareil photographique qui traduit le mieux la violence à laquelle il assiste et participe. Il voit les paysages dévastés avant les morts, avec la froideur de celui qui ne peut que montrer parce qu'il ne parvient pas à dire. L'histoire du regard photographique change de visage avec le premier conflit mondial. Pour plusieurs décennies, les pupilles masculines portent la marque de ce qu'elles ont vu, et la société toute entière ne peut ignorer les blessés, dont le spectacle quotidien, frisant parfois l'insupportable, rappelle sans cesse que rien ne sera plus jamais comme avant. ${ }^{44}$

\footnotetext{
${ }^{42}$ Frédéric CHAUVAUD, De Pierre Rivière à Landru. La violence apprivoisée au XIXe siècle, Bruxelles, Brepols, 1991, pp. 257-259.

${ }^{43}$ Stéphane AUDOIN-ROUZEAU, La guerre des enfants. 1914-1918. Essai d'histoire culturelle, Paris, Armand Colin, 1993, pp. 9-11.

${ }^{44}$ Sophie DELAPORTE, Les gueules cassées. Les blessés de la face de la Grande Guerre, Paris, Noêsis, 1996, $231 \mathrm{p}$.
} 